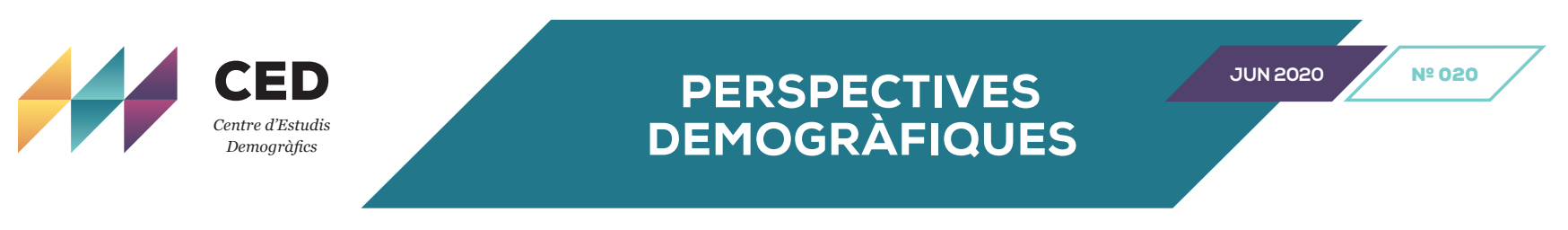

\title{
Covid-19: ¿la vivienda protege a los mayores en América Latina? Argentina y Colombia comparadas con España
}

Juan A. Módenes, Centre d'Estudis Demogràfics y Depto. de Geografía, Universitat Autònoma de Barcelona (ESP), Mariana Marcos, Univervidad de Buenos Aires, Consejo de Investigaciones Científicas y Técnicas y Universidad Nacional de Tres de Febrero (ARG) y Diva Marcela García, Pontificia Universidad Javeriana y Universidad Externado de Colombia $(\mathrm{COL})$

Proteger a la población mayor en el nuevo paradigma de la covid-19 no puede confiarse solo al confinamiento en la vivienda. Menos aún en América Latina. La estructura de los hogares, las características de la vivienda y la actividad laboral de los corresidentes condicionan la exposición de los mayores a los contagios dentro de la vivienda. Para medir esa exposición, este número de Perspectives Demogràfiques propone el cálculo de un indicador que estima el riesgo residencial multidimensional, y que permite la comparación entre poblaciones. Más de la mitad de las personas mayores de Colombia y un 35\% de las de Argentina están sobreexpuestas a la covid-19 en casa. Nuestro estudio, al demostrar que el riesgo residencial es muy importante en América Latina, pero también significativo en España, evidencia que minimizarlo debe ser un objetivo prioritario en la protección de las personas mayores. El indicador de riesgo residencial ante el covid-19 puede utilizarse para identificar áreas o subpoblaciones más expuestas dentro de los países y guiar la acción política futura ante la pandemia.

\section{LAS PERSONAS MAYORES FRENTE A LA COVID-19 EN AMÉRICA LATINA}

La covid-19 ha impactado de lleno en la población mayor. Constituye el principal grupo de riesgo al tener una sintomatología más grave y una letalidad mucho más alta. Por lo que respecta a América Latina, la ventaja del moderado envejecimiento poblacional de la región se verá negativamente compensada por la mayor prevalencia de enfermedades crónicas y a edades más jóvenes, la desigualdad, las menores posibilidades de distanciamiento social (Acosta, 2020) y la concentración espacial de población en grandes ciudades con altos niveles de segregación.

Es primordial proteger a los mayores tanto en la actual como en futuras oleadas, mientras no se obtenga una curación efectiva (Huenchuan, 2020). Esta protección debe consistir en contener el contagio primario, pero sobre todo en disminuir los contagios secundarios por transmisión intrafamiliar dentro de las viviendas (Esteve et al, 2020; Mogi, Spijker 2020). En América Latina las muertes por contagio secundario podrían duplicar las producidas por contacto primario (Esteve et al, 2020). Mientras que en Europa la enfermedad ha afectado con mayor intensidad a los mayores institucionalizados, manifestando los problemas de este modelo de cuidado (ComasHerrera, Zalakain, 2020), en América Latina los mayores viven menos en instituciones, pero suelen corresidir en estructuras familiares complejas, de carácter intergeneracional (Saad, 2005). En ellas, las personas difícilmente pueden aislarse por completo, a lo que se suman los riesgos aportados por los corresidentes que deben salir a trabajar, a

FIGURA 1. Personas de 65 y más años según riesgo residencial ante la covid-19. Colombia, Argentina y España

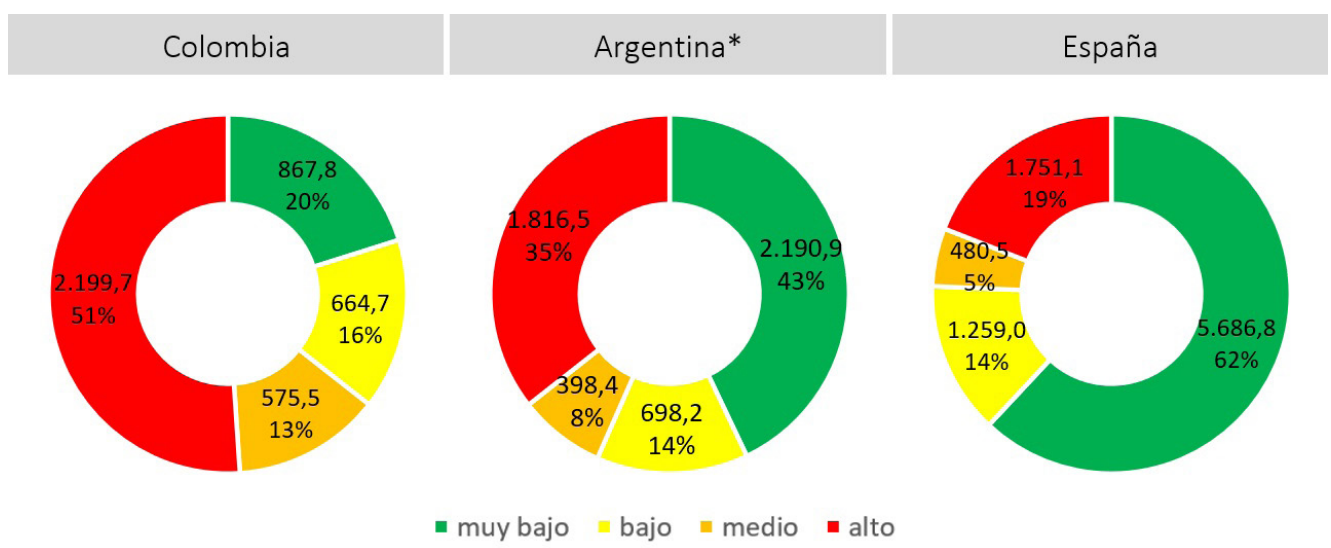

Fuente: Elaboración propia a partir de Gran Encuesta Integrada de Hogares 2019 (DANE, Colombia), Encuesta Permanente de Hogares $1^{\circ}$ semestre 2019 y proyecciones 2010 (INDEC, Argentina) y Encuesta de Condiciones de Vida 2018 (INE, España)

*Encuesta representativa del 73\% de los adultos mayores. Absolutos estimados en base a proyecciones 
menudo en condiciones de informalidad, precariedad y con escasas posibilidades de teletrabajo.

La fuerte desigualdad social de América Latina agrava para muchos los riesgos de contraer la covid-19 (CEPAL, 2020). Los riesgos residenciales que entrañan los hogares de gran tamaño, la convivencia intergeneracional, la mala calidad de la vivienda, la necesidad de trabajar de los mayores y la ocupación informal, precaria y de baja calificación de los corresidentes, se intensifican en los grupos socioeconómicos más bajos. Avanzar en la lucha contra la enfermedad implicará priorizar la protección de las personas mayores en entornos de vulnerabilidad social. Y ello obliga a evaluar la situación de partida de esta población en América Latina y las desigualdades en diferentes escalas. De ahí nuestro interés en contrastar dos casos representativos y distintos, Colombia y Argentina. Para acabar de comprender y contextualizar los resultados los compararemos con los de España.

En este trabajo inicial, aportamos un método que permite sintetizar los riesgos de contagio en la vivienda. Los resultados más generales (figura 1) son muy reveladores. Mientras en Colombia el 51\% de los mayores están sometidos a un riesgo residencial alto ante la covid-19, este porcentaje baja a un $35 \%$ en Argentina y hasta un 19\% en España. Por otro lado, solo un $21 \%$ de los mayores colombianos tendrían un riesgo muy bajo, siendo del 42\% en Argentina y del 62\% en España.

FIGURA 2. Definición de los niveles de riesgo residencial ante la covid-19 en función de condiciones de corresidencia, vivienda y laborales

\begin{tabular}{|c|c|c|c|}
\hline $\begin{array}{l}\text { Tipo de hogar y } \\
\text { allegamiento } \\
\text { externo }\end{array}$ & $\begin{array}{l}\text { Condiciones de } \\
\text { riesgo de la } \\
\text { vivienda }\end{array}$ & $\begin{array}{l}\text { Probabilidad de que } \\
\text { algún residente no } \\
\text { pueda teletrabajar }\end{array}$ & \multirow[t]{17}{*}{$\begin{array}{l}\text { Condiciones de } \\
\text { riesgo residencial } \\
\text { ante la covid-19 }\end{array}$} \\
\hline \multirow{8}{*}{$\begin{array}{l}\text { unipersonal o } \\
\text { pareja sola, } \\
\text { único hogar } \\
\text { en la vivienda }\end{array}$} & sin hacinamiento & nadie trabaja & \\
\hline & por cuarto y con & baja & \\
\hline & agua de red en & media & \\
\hline & la vivienda & alta & \\
\hline & con hacinamiento & nadie trabaja & \\
\hline & por cuarto o sin agua & baja & \\
\hline & de red en & media & \\
\hline & la vivienda & alta & \\
\hline \multirow{8}{*}{$\begin{array}{l}\text { otro arreglo } \\
\text { residencial }\end{array}$} & sin hacinamiento & nadie trabaja & \\
\hline & por cuarto y con & baja & \\
\hline & agua de red en & media & \\
\hline & la vivienda & alta & \\
\hline & con hacinamiento & nadie trabaja & \\
\hline & por cuarto o sin agua & baja & \\
\hline & de red en & media & \\
\hline & la vivienda & alta & \\
\hline
\end{tabular}

Fuente: Elaboración propia

\section{MEDIR EL RIESGO RESIDENCIAL}

Nuestro indicador permite cuantificar el riesgo de tener contacto doméstico con la enfermedad covid-19. Se ha calculado a partir de una serie de dimensiones familiares, residenciales y laborales relacionadas con la protección ofrecida por la vivienda a las personas mayores (figura 2). Es lo que llamamos riesgo residencial. Para ello, en primer lugar, se mide la distribución de personas mayores según conviven en hogares unipersonales y en pareja sola (menor riesgo) o con otras personas (más riesgo). En segundo lugar, se estima si el hogar reside en su vivienda en condiciones de hacinamiento (más de 2 personas de media por dormitorio, más riesgo) y déficit de suministros esenciales (sin agua corriente, más riesgo) o no -dimensión especialmente relevante en América Latina-. En tercer lugar, se identifica si existen sujetos que trabajan en la vivienda incluyendo las personas mayores (más riesgo) o no. Y, para completar esta tercera dimensión, consideramos hasta qué punto los trabajos de los miembros del hogar les obligan a salir de la vivienda (más riesgo) o se pueden desarrollar en forma de teletrabajo (posiciones sociales más altas y de menor riesgo). El riesgo residencial total será más elevado cuantas más dimensiones individuales de riesgo se combinen.

Como fuentes, se han empleado encuestas sociodemográficas de los tres países que cumplen los requisitos de ser actuales, comparables e integrar las dimensiones comentadas. En el caso de Colombia se ha utilizado la Gran Encuesta Integrada de Hogares de 2019, para Argentina las fuentes son la Encuesta Permanente de Hogares del 1er semestre 2019 y las proyecciones de población 2010 y de España se ha explotado la Encuesta de Condiciones de Vida de 2019. El universo comprende a las personas de 65 y más años de edad en el momento de las encuestas, que viven en hogares particulares.

Una tarea importante ha sido estimar la probabilidad de que una persona ocupada pueda teletrabajar o, por el contrario, esté obligada a salir de la vivienda. Nos hemos basado en Bonaviday Gasparini (2020) que han estudiado la probabilidad de que cada ocupación (2 dígitos, código internacional CIUO) pudiese efectuarse en modalidad teletrabajo. Hemos aplicado sus resultados a 
los tres países para calcular la probabilidad compuesta de que en una unidad de corresidencia alguna persona tuviera que salir fuera a trabajar. Esta probabilidad tiende a ser mayor cuantos más trabajadores conviven en la vivienda, por lo que también está asociada positivamente al tamaño del grupo doméstico.

Al combinar todas las dimensiones se ha establecido una parametrización jerárquica del riesgo experimentado por los mayores en su vivienda. La figura 2 presenta esta "semaforización" del riesgo. Si la persona mayor vive sola o con su pareja sin el agregado de otras personas u hogares en la vivienda, sus condiciones de vivienda son adecuadas y nadie trabaja en la vivienda, el riesgo es muy bajo. Obviamente siempre habrá un riesgo de contagio, sea por infección primaria al principio de un brote, o sea porque en confinamiento o en otras etapas de control, la reclusión no suele ser absoluta. Actividades puntuales en la calle, como comprar víveres, pueden todavía significar un cierto riesgo. En el otro extremo, el riesgo es alto cuando la persona mayor vive con otras personas, en particular con alguien que deba trabajar fuera del domicilio, o si ella misma debe hacerlo, y el hogar está hacinado o no dispone de agua corriente en la vivienda.
Según datos de cada una de las dimensiones del riesgo residencial (figura 3), en Colombia el 67\% de las personas mayores comparten su vivienda con alguien distinto a su pareja. Tan sólo el 38\% reside en hogares donde nadie trabaja y en un $52 \%$ comparte su vivienda con alguien que muy probablemente debe salir a trabajar. Además, un 14,6\% de los mayores colombianos radican en viviendas con hacinamiento o déficit del servicio de agua. En Argentina, en cambio, los riesgos residenciales son menores, pero también importantes: un $43 \%$ de los mayores comparte su vivienda con personas que no son su pareja y solo un $54 \%$ de los mayores viven en un hogar donde nadie trabaja. Un 35\% de los mayores argentinos convive con alguien que es muy probable que se vea obligado a trasladarse para trabajar. $\mathrm{Y}$ en un $5 \%$ de casos la vivienda sufre de hacinamiento o no recibe agua corriente dentro de la vivienda.

En España todos los parámetros en términos relativos son más favorables. Casi dos tercios de los mayores moran en hogares en solitario o en pareja, en casi el cien por cien de los casos sin hacinamiento. Por otro lado, un $75 \%$ de los mayores residen en hogares donde nadie trabaja; un $80 \%$ en hogares donde es poco probable que alguien tenga que ir a trabajar fuera de la vivienda.
FIGURA 3. Personas de 65 y más según condiciones de corresidencia, vivienda y laborales, Colombia, Argentina y España

Dimensión de riesgo
Riesgo del hogar
no es unipersonal/ pareja sola,
o hay más de un hogar
" unipersonal o pareja sola,
único hogar en la vivienda
- otro arreglo residencial
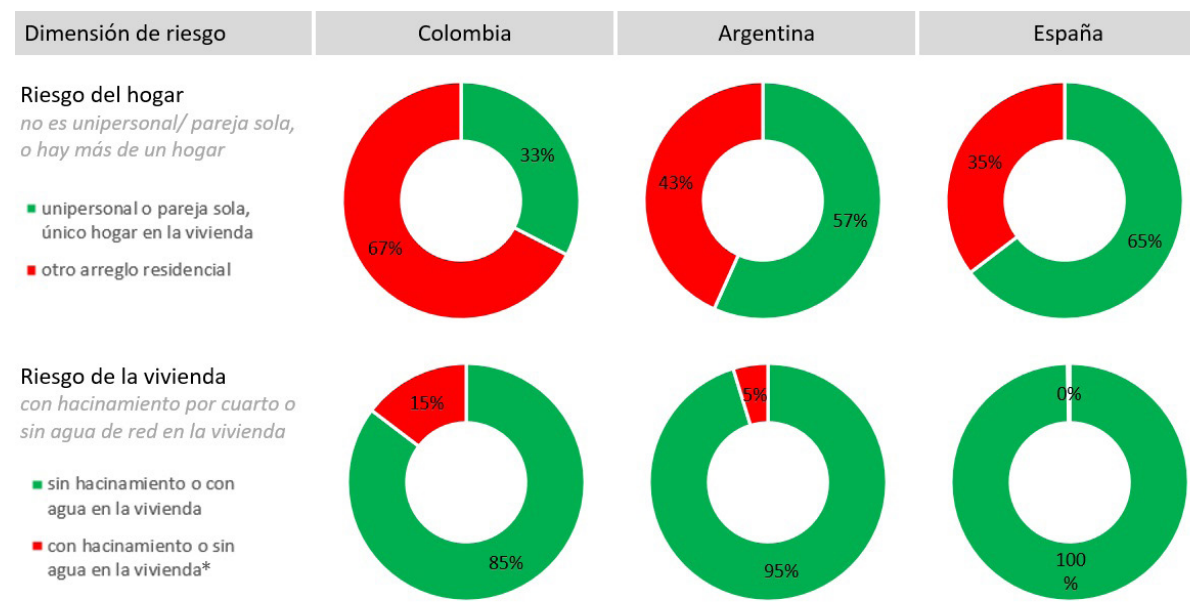

Riesgo por activ. laboral hay ocupados en la vivienda con altas probabilidades de no poder teletrabajar

- nadie trabaja = riesgo medio

riesgo bajo - riesgo alto

= hay ocupado(s) sin datos**
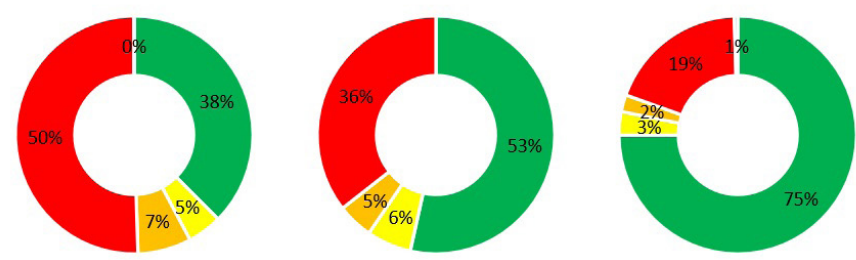

Fuente: Elaboración propia a partir de Gran Encuesta Integrada de Hogares 2019 (DANE, Colombia), Encuesta Permanente de Hogares $1^{\circ}$ semestre 2019 (INDEC, Argentina) y Encuesta de Condiciones de Vida 2018 (INE, España)

* Argentina: incluye personas en villa

** Argentina: en ausencia de datos de ocupación, riesgo por no poder teletrabajar imputado a partir de la rama de actividad
Los resultados apuntan varias líneas. En primer lugar, que en América Latina la frecuente corresidencia intergeneracional es decisiva para calcular el riesgo. En España la extendida autonomía residencial de las personas mayores puede actuar como un factor protector del contagio dentro de la vivienda. En América Latina, en cambio, el grado de autonomía residencial se relaciona con la estructura social. En segundo lugar, cuando los mayores conviven con personas más jóvenes, el riesgo relacionado con la actividad laboral aumenta. Y ello se agrava cuando es la persona mayor misma la que debe trabajar (en ausencia de protección de empleo formal o prestaciones adecuadas por jubilación).

Aunque las condiciones en España son mejores y su población total similar a la de Argentina y Colombia, su mayor envejecimiento eleva considerablemente las cifras absolutas de mayores en riesgo 
residencial. Así mientras que en Colombia viven en riesgo alto 2 millones de personas de 65 y más años, en Argentina se estima que son 1,8 millones y en España 1,7 millones. El riesgo de que los ancianos estén en contacto dentro de la vivienda con la enfermedad es elevado en los tres países considerados. No es algo puntual sino estructural.

\section{PROTEGER A LOS MAYORES}

Hemos mostrado que no es buena estrategia confiar solo en la eficacia del confinamiento para proteger a los grupos con un riesgo superior de la población. Los riesgos residenciales de las personas mayores no son despreciables en ningún contexto, pero son mucho más elevados en América Latina. Los contactos cotidianos entre las personas, allegadas o no, son multidimensionales y difíciles de evitar del todo, cuando los mayores no viven solos o en pareja. En este estudio hemos abordado algunas dimensiones críticas de este contacto social: la convivencia en la vivienda, su calidad y la necesidad de realizar la actividad laboral fuera del hogar. Y, concretamente, hemos propuesto un indicador de riesgo residencial a partir de variables que miden las dimensiones anteriores, y expresa la vulnerabilidad de los mayores en su contexto doméstico. Lo hemos aplicado a Colombia y Argentina, que muestran la especificidad de América Latina en comparación con España, un país europeo, y, a la vez, la heterogeneidad interna de la región.

Aunque la población anciana no suele vivir hacinada, la incidencia de este problema (aun cuando sea baja) basta para dificultar los confinamientos generales obligatorios o la eventual

\section{Referencias bibliográficas}

Acosta, E. (2020) Seminario Virtual "Epidemias en América Latina: historia y presente". Programa de Población, Facultad de Ciencias Sociales, Udelar, 12-5-2020.

Bonavida, C.; Gasparini, L. (2020) "El Impacto Asimétrico de la Cuarentena”. Documentos de Trabajo del CEDLAS, 261, CEDLAS-FCEUNLP (http://www.cedlas.econo.unlp.edu.ar/ wp/no-261/).

CEPAL (2020) El desafío social en tiempos del covid-19. Informe especial (https://repositorio.cepal.org/handle/11362/45527)

Comas-Herrera A.; Zalakain J. (2020) Mortality associated with COVID-19 outbreaks in care homes: early international evidence. LTCcovid.org, International Long-Term Care Policy Network, CPEC-LSE, April (https:// ltccovid.org/wp-content/uploads/2020/04/ Mortality-associated-with-COVID-12-April-2 pdf).

Esteve, A.; Permanyer, I.; Boertien, D; Vaupel, J. (2020) "National age and co-residence patterns shape covid-19 vulnerability". medRxiv (https://doi.org/10.1101/2020.05.13.201002 89). necesidad de aislamiento individual. En América Latina la débil protección social también obliga a muchas personas mayores a continuar trabajando, a menudo en ocupaciones de baja calificación y en condiciones de informalidad. Si no es el caso, la corresidencia con personas más jóvenes y otros hogares las pone frecuentemente en contacto con quienes sí deben y necesitan salir a trabajar. Pautas complejas de corresidencia están asociadas a situaciones laborales del hogar más precarias y mayores dificultades para minimizar el contacto social. En definitiva, cualquier medida de protección de los mayores debe anticipar que aislarlos completamente de personas de menos riesgo, pero mayor exposición es poco realista y, no pocas veces, contraproducente. Además, los resultados muestran que las situaciones intermedias de riesgo son minoritarias, por lo que existe una cierta polarización del riesgo residencial que habrá que considerar.

Nuestro método consigue detallar los riesgos residenciales por otras variables relevantes, como la edad, el sexo, los grupos sociales y los niveles geográficos. Replicado en base a datos censales, también añade el potencial de identificar las áreas donde se concentran más estos riesgos, en relación con la gran heterogeneidad de las estructuras sociodemográficas y residenciales de América Latina. Al mismo tiempo, resulta fundamental, a más largo plazo, para planificar futuros escenarios de protección de este $u$ otros grupos de riesgo en la eventualidad de nuevos episodios de transmisión de enfermedades infecciosas. Y finalmente permite la evaluación comparativa de estos nuevos retos a los que nos enfrentamos.

Editores
Andreu Domingo y Albert Esteve
(Centre d'Estudis Demogràfics)
Correspondencia dirigidida a
Juan A. Módenes
jamodenes@ced.uab.es
Mariana Marcos
mmarcos@conicet.gov.ar
Diva Marcela García
diva.garcia@javeriana.edu.co
Agradecimientos
Este informe se inscribe en los proyectos
I+D+I "Comportamientos demográficos
y estrategias residenciales: apuntes para
el desarrollo de nuevas políticas sociales"
(CSO2016-79142-R, España) y "Desigual-
dades microespaciales urbanas" (32/375 A,
UNTREF, Argentina). Se agradece al Centre
d'Estudis Demogràfics (CERCA Programme /
Generalitat de Catalunya) por su apoyo, espe-
cialmente a su responsable del banco de datos
Tere Menacho. La autoría también agradece
la ayuda de Maru Fernández, Jimena Johan,
Florencia Ansaloni y Alejandra Hernández.

Créditos

Gráficos: Mariana Marcos Maquetación: Xavier Ruiz Vilchez

chuan S. (2020) Covid 19: recom daciones generales para la atención a personas mayores desde una perspectiva de derechos humanos. México: CEPAL (https:// repositorio.cepal.org/bitstream/handle/11362/45316/4/S2000271_es.pdf).

Mogi, R.; Spijker, J. (2020) "The influence of social and economic ties to the spread of COVID-19 in Europe". SocArXiv (https://doi org/10.31235/osf.io/sb8xn).

Saad, P. (2005) "Los adultos mayores en América Latina y el Caribe: Arreglos residenciales y transferencias informales". Notas de Población, 80: 127-154 (https://repositorio.cepal. org//handle/11362/12970).

Cita

Juan A. Módenes, Mariana Marcos, Diva Marcela García (2020) "Covid-19: ćla vivienda protege a los mayores en América Latina? Argentina y Colombia comparadas con España". Perspectives Demogràfiques, 20: 1-4 (ISSN: 2696-4228).

ISSN

Enlace url

http://ced.uab.es/es/difusion/butlletiperspectives-demografiques

Contacto

Centre d'Estudis Demogràfics. Calle de Ca n'Altayó, Edificio E2 Universitat Autònoma de Barcelona 08193 Bellaterra / Barcelona España

Teléfono: +34935813060 E-mail: demog@ced.uab.es Web: http://ced.uab.es/es/

ISSN 2696-4228 